\title{
Sozialen Sinn verstehen durch rekonstruktive Genogrammarbeit
}

Andrea Heistinger

Im sinnverstehenden Ansatz der Genogrammarbeit geht es um Interaktion, um Verbindung, um das Sichtbarmachen von WechselWirkungen zwischen Makrosoziologie - großen gesellschaftlichen Entwicklungen - und der individuellen Biografie eines Menschen oder einer Familie - der Mikrosoziologie. In anderen Worten: Es geht um Beziehung. Konkret fokussiert die rekonstruktive Genogrammarbeit auf historischen und familiensoziologischen Daten der letzten drei Generationen eines individuellen Menschen. In unserem Fall waren dies je die vier Großeltern und zwei Eltern der beiden Betriebsleiter*innen. Dabei geht die rekonstruktive, ressourcenorientierte Genogrammarbeit davon aus, dass aus der Vergangenheit keine kausalen oder linearen Linien in eine Zukunft hineingezogen werden können. Vielmehr bildet die Vergangenheit einen Rahmen, der mögliche Entwicklungen offen hält und der auch überschritten werden kann. ${ }^{1}$

\section{Eine kurze Theorie der Genogrammarbeit}

\section{Genogrammarbeit versteht Sinn als sozialer Sinn}

Der Genogrammarbeit nach Bruno Hildenbrand liegt die objektive Hermeneutik als Sichtweise des Verstehens menschlichen Verhaltens zu- 
grunde. Sie hat sich als spezifische Variante der objektiven hermeneutischen Fallrekonstruktion entwickelt. ${ }^{2}$ Sie geht davon aus, dass Werke objektivierte Gegenstände sind, die auf menschliches Handeln zurückgehen. ${ }^{3}$ Mit anderen Worten: Im »Werk « manifestiert sich ein Objekt, das von einem Subjekt geschaffen wurde. Menschen schaffen Werke und realisieren sich darin als Menschen - und zwar in Auseinandersetzung und in Beziehung mit anderen Menschen. Die Genogrammarbeit knüpft hier an und versteht das Genogramm eines Menschen als Werk. ${ }^{4}$

Die Frage ist nun, wie regionale Muster der Daseinsbewältigung ${ }^{5}-$ die ja kollektive Muster sind - mit den individuellen Momenten im Leben eines Menschen (den objektiven Daten in seinem Genogramm) verbunden sind. Hier knüpft Hildenbrand an das Menschenbild des französischen Soziologen Pierre Bourdieu an und zwar konkret an dessen Begriffe Habitus und Praktiken.

In unserem Ansatz der sorgsamen Landwirtschaft gehen wir darüber hinaus und auch davon aus, dass Menschen sich in den von ihnen geschaffenen Werken auch durch ihre Auseinandersetzung mit der $\mathrm{Na}$ tur, mit den Tieren, die sie halten, mit den Pflanzen, die sie anbauen, mit den Äckern, die sie kultivieren, realisieren. In der Landwirtschaft

2 Unter Objektiver Hermeneutik werden Interpretationsverfahren der empirischen Sozialforschung verstanden, die im Wesentlichen auf den Soziologen und Sozialpsychologen Ulrich Oevermann zurückgehen. Dabei gibt es nicht die objektive Hermeneutik, sondern ein gemeinsames Grundverständnis für die Herangehensweise an die Deutung von Daten. (Vgl. Kruse 2015: 417).

3 Hildenbrand 2013:93.

4 Hildenbrand 2013, ebenda.

5 Für die Forschungsregion Südoststeiermark beschreiben die Kulturanthropologen Karl Kaser und Karl Stocker dies so: »In den einzelnen Dörfern hatten sich im Laufe der Jahrhunderte >ungeschriebene Gesetze< entwickelt, die das Leben der BewohnerInnen regelten. Mochten sie auch für so manchen Außenstehenden unverständlich sein, für die südoststeirische bäuerliche Bevölkerung waren sie durchaus leicht erfassbar. Ob es sich um Richtlinien für die Arbeit (so galt es etwa >fleißig`zu sein), um Regeln für die lokalen sozialen Beziehungen (...) oder um die Haltung zur Kirche handelte, niemand zweifelte diese sungeschriebenen< Gesetze an.« Kaser u.a. 2003:307f. 
kann das Objekt ein Hof sein, aber auch die angebauten Pflanzenarten, die gehaltenen Tierrassen, der auf eine bestimmte Art hergestellte Käse, ein verwendetes Gerät, die über die Praktiken und Vorstellungen der Menschen, die einen Betrieb bewirtschaften, verbunden sind. ${ }^{6}$

\section{Handeln und Familienmuster}

Familienmuster prägen latent die Entscheidungen, die in der Familie getroffen werden. ${ }^{7}$ Muster, die aus der vollzogenen Lebenspraxis der Vergangenheit stammen, geben einerseits Dynamiken - im Sinne von Handlungsorientierungen - vor, andererseits können sie sich laufend wandeln. Und genau das ist entscheidend: Familienmuster sind immer veränderbar. Dieser Fokus ist sowohl für die Forschung, wie auch für die Beratung essentiell. ${ }^{8}$ Familienmuster sind wie kognitive - also gedachte - Landkarten mit deren Hilfe sich ein System, in unserem Fall ein Familiensystem, in seiner Wirklichkeit orientiert. Diese Orientierungsstrukturen betreffen "nicht nur die kognitive Ebene, sondern gleichermaßen emotionale sowie vor-kognitive Muster alltagsweltlicher Orientierung. «9 Familienmuster haben kollektive Anteile, die für die Zeit und die Region typisch sind und Anteile, die für die Familie typisch sind. Das Individuelle einer Geschichte ist stets in kollektive Handlungsmuster eingewoben. Bruno Hildenbrand beschreibt dies so: "Akteure arbeiten sich interpretierend am Vorgegebenen (»objektive Daten «) ab und transformieren das Vorgegebene in ein Aufgegebenes, das heißt zu Gestaltendes, indem sie unter objektiv gegebenen Möglichkeiten auswählen. ${ }^{10}$

6

Siehe ausführlich das Kapitel von Gabriele Sorgo sowie das Kapitel Wie aus Familiengeschichte sorgsame Landwirtschaft entsteht.

7 Hildenbrand 2018:20.

8 Auch die Agrarsoziologin Ika Darnhofer legt in ihren Forschungen zur Resilienz von Familienbetrieben den Fokus auf die Frage, welche Beziehungsmuster (i.O. »patterns of relations«) es sind, die einen transformativen Wandel ermöglichen. Darnhofer u.a. 2016.

9 Welter-Enderlin und Hildenbrand 2004:21.

10 Hildenbrand 2005:23. 


\section{Die Praxis der Genogrammarbeit - Die einzelnen Arbeitsschritte der Genogrammarbeit im Forschungskontext}

\section{Zur Fallauswahl - Theoretisches Sampling von Fall zu Fall}

Für die Fallauswahl wurde keine Stichprobe gewählt, sondern das theoretische Sampling erfolgte von Fall zu Fall. Diese Methode folgt dem von Hildenbrand vorgeschlagenen Weg, nicht alle Familienstudien (= Fallbeispiele) zu Beginn des Forschungsprozesses auszuwählen, sondern zunächst den ersten Fall, diesen durch die sequentielle Interpretation $\mathrm{zu}$ erschließen und nach Formulierung einer Fallstrukturhypothese zu den Familienmustern des ersten Falls den zweiten Fall in einer maximalen Fallkontrastierung zum ersten Fall auszuwählen. ${ }^{11}$ Im Forschungsprojekt haben wir die Methode dahingehend abgewandelt, dass wir nicht nach Familienmustern kontrastiert haben, sondern nach betrieblichen Daten - die wiederum als objektive Werke in der Biografie einer Familie verstanden werden. Nachdem der erste Betrieb ein neu gegründeter Betrieb war, haben wir als zweiten Fall einen Betrieb ausgewählt, der in der Familie nach patrilinearer Vererbungspraxis übergeben wurde und in dem das Betriebsleiterpaar eine neue Wirtschaftsweise eingeführt hat. Als dritten Betrieb einen Tierhaltungsbetrieb mit Direktvermarktung, der von einem Ehepaar neu gegründet worden war und als vierten Fall wiederum einen spezialisierten Betrieb, der von der dritten Generation (unseren Interviewpartner*innen) vom Hobbybetrieb in einen Haupterwerbsbetrieb umgewandelt wurde.

\section{Daten erheben}

Die Genogrammdaten wurden im Gespräch mit den Betriebsleiter*innen direkt bei den Besuchen auf den Betrieben während der Feldforschung erhoben. Diese Daten wurden von den Gesprächspartner*innen

11 Hildenbrand 1999. Siehe dazu ausführlich das Kapitel »Wie aus Familiengeschichten sorgsame Landwirtschaft entsteht«. 
entweder spontan erinnert, fehlende Daten haben die Gesprächspartner*innen auf unsere Bitte hin nachrecherchiert und uns nachträglich per E-Mail übermittelt. In der Regel werden die Genogrammdaten der letzten drei Generationen einer Person erhoben (also des/der Betriebsleiter*innen - auch der ehemaligen Partner*innen, falls diese auch für den Betrieb relevant waren, Geschwister, Eltern und Tanten/Onkeln sowie Großeltern/wenn möglich auch Großtanten und Großonkeln); in unserem Fall von allen Betriebsleiter"innen, inklusive ehemaliger Partner*innen. Insbesondere interessieren jene Entscheidungsbereiche, die für die Identitätsbildung und eine autonome Lebenspraxis entscheidend sind: Die Entscheidung für einen Beruf (die materielle Selbsterhaltung), die Partner*innenwahl und die Entscheidung für oder gegen Kinder und die Herstellung eines Bezugs zum Gemeinwesen (Wahl des Wohnorts, Beziehungen zu Institutionen). ${ }^{12}$ Erhoben werden ausschließlich objektive Daten. ${ }^{13}$ Der Vergleich der spontan erinnerten mit den nachträglich von den Interviewpartner*innen recherchierten Daten trägt zum Fallverstehen und zur Rekonstruktion des Familienmusters bei. Folgende Daten werden - im optimalen Fall der Vollständigkeit von Daten - erhoben:

- Vorname(n) und Nachname

- Geburtsjahr und allenfalls Sterbejahr

- Partnerschaften/Eheschließungen

- Allenfalls Trennung oder Scheidung (Jahr)

- Schulbildung und allenfalls weitere Ausbildungen

- Ausgeübte(r) Beruf(e)

12 Siehe dazu ausführlich Hildenbrand 2005:19.

13 Wir haben bis auf eine Ausnahme, wo wir Genogrammdaten auch im Gespräch mit der Mutter eines Betriebsleiters erhoben haben, nicht mit den Eltern, Onkeln, Tanten, Großeltern oder Geschwistern, persönlich gesprochen. Die Interviewpartner*innen sind unsere Informant*innen zu allen anderen Personen, lebend oder verstorben - es sind also ihre erinnerten oder preisgegebenen Daten sowie Daten, die unsere Interviewpartner*innen selbst noch innerhalb der Familie nachrecherchiert haben und die manchmal auch die im Gespräch spontan erinnerten Daten revidiert haben. 
- Wohnort(e) und Wohnortwechsel

- In günstigen Fällen auch Daten zu Zeiten der Beschäftigung/Arbeitslosigkeit/Militär- oder Zivildienst und Pensionierung

- Karenzzeiten

Die Vollständigkeit der neun im Forschungsprojekt erhobenen Genogramme ist unterschiedlich. Zum Teil mussten wir auch mit unvollständigen Genogrammen arbeiten, weil Genogramme nicht genau erinnert wurden oder unsere Gesprächspartner*innen nicht alle Daten nachrecherchieren konnten, weil beispielsweise der Kontakt zu einem Familienmitglied abgebrochen war.

\section{Im Team das Genogramm sequentiell rekonstruieren und Familienmuster beschreiben}

Im Verständnis von Hildenbrand werden Familienmuster nicht in die Genogrammdaten hineingedeutet, sondern aus ihnen herausgelesen. Ein wesentliches Element der Methode der Genogrammarbeit ist es, die Entscheidungen, die einzelne und ihr Familien getroffen haben, sowie die Spuren, die sie in der sozialen Wirklichkeit hinterlassen haben, zu rekonstruieren. ${ }^{14}$ Die rekonstruktive Genogrammarbeit ist daher - wie in der objektiven Hermeneutik üblich - eine Sequenz-Analyse. ${ }^{15}$ Man fängt bei einem Großvater oder einer Großmutter der »Indexperson « in unserem Falle der Betriebsleiter oder die Betriebsleiterin - an und notiert seinen/ihren Namen, Geburtsort, Geburtsjahr und Beruf auf ein Flipchart. Nun geht es darum, an jeder biografischen Entscheidungssequenz die begründbaren Handlungsmöglichkeiten zu rekonstruieren und erst in einem zweiten Schritt die im Einzelfall tatsächlich gewählte Option offenzulegen. Am einfachsten ist dies, wenn die Person, die das

\footnotetext{
14 Hildenbrand 2013.

15 Die theoretische Grundlage der Sequenzanalyse ist: Die Lebenspraxis als Prozess der Krisenbewältigung zu sehen. Welter Enderlin und Hildenbrand 2004:32.
} 
Genogramm erhoben hat, zunächst nur zuhört und die anderen Personen der Arbeitsgruppe - oder des Arbeitstreffens - Hypothesen formulieren. Dazu ist ein sozioökonomisches und historisches Hintergrundwissen zur Region, in der der Betrieb liegt, und zu den agrarpolitischen Rahmenbedingungen der jeweiligen Zeit notwendig und es ist hilfreich, wenn ein Team von mehreren Forscher*innen am Deutungsprozess beteiligt ist. Was waren zu diesem Zeitpunkt mögliche Optionen für die Großmutter, den Großvater, den Vater und die Mutter des/der Betriebsleiter"in? Man beginnt also jeweils mit der Großelterngeneration. Jedes Datum, jede Angabe wird im Forschungsteam zunächst so besprochen, indem nach allgemeinen, (damals) denkbaren Handlungsoptionen gefragt wird. Dieser Schritt ist ein kontextfreies Analysieren - der unmittelbare, spezielle Kontext der Fallgeschichte wird also zunächst bewusst ausgeblendet. ${ }^{16}$ Erst nachdem wir die objektiv möglichen Handlungsmuster benannt haben, wird aufgedeckt, wie die konkrete Person tatsächlich gehandelt hat. Von hier aus befassen wir uns mit den tatsächlich eingetretenen Möglichkeiten, bis wir in der Gegenwart angekommen sind und verdichten so die Arbeitsthesen zu Familienmustern.

Damit das gelingt, werden Genogrammdaten konsequent von den subjektiven Erzählungen in der Familie getrennt. Auch und gerade dann, wenn es sich um in der Familie tradierte Erzählungen handelt. Das Genogramm wird nicht vollständig im Team präsentiert, sondern im Team gemeinsam Schritt-für-Schritt rekonstruiert. Konkret haben wir zur Rekonstruktion eines Genogramms zwischen zwei bis vier Stunden verwendet. Auf diese Weise ist nicht nur das Interpretationsverfahren sehr entschleunigt, was die Perspektive der Forscher*innen öffnet und ein Grundprinzip im Deutungsprozess rekonstruktiv-hermeneutischer Verfahren entspricht, ${ }^{17}$ sondern die

16 Auch dieses bewusste Ausblenden erfordert Übung. Und da wir ja die Cenogramme der beiden, den Betrieb bewirtschaftenden Partner*innen erhoben haben, war bei der Interpretation des jeweils zweiten Genogramms einiges bereits bekannt. Für die Benennung von möglichen Handlungsoptionen war dies allerdings nicht hinderlich. 
Person, die gerade im Fokus der Genogrammarbeit steht, wird in ihren jeweiligen Rahmenbedingungen und Handlungsmöglichkeiten - ähnlich wie eine Rolle in einem Theaterstück - sicht- und wahrnehmbar. In der rekonstruktiven Genogrammarbeit werden so Schritt für Schritt die Lebens- und Familienthemen der letzten drei Generationen zu einer Fallstrukturhypothese verdichtet. Diese Form der Rekonstruktion von Genogrammen hat auch eine durchwegs heitere und spannende Komponente. Je nachdem, wie umfangreich das Wissen über die Lebensverhältnisse $\mathrm{zu}$ dieser Zeit und an diesem Ort sind, können detailreichere Thesen formuliert werden.

Entscheidungen, die aufs erste als individuelle unternehmerische Entscheidungen erscheinen, stehen häufig auch in einer Interaktion mit Dynamiken und Handlungsmustern innerhalb der Familie. Dazu ein Fallbeispiel aus unserer Forschung: Die Entscheidung des Ehepaars Bergmanns, die Intensiv-Hühnermast aufzugeben und den Betrieb auf einen CSA-Gemüsebetrieb umzustellen scheint aufs erste ein radikaler Bruch mit der Geschichte des Betriebs zu sein. Bei einer sequentiellen Analyse des Genogramms der Eltern und Großeltern von Herrn Bergmann wird sichtbar, dass auch sie sich jeweils als erste in der Region auf radikal Neues einlassen konnten - den Anbau damals neuer Kulturen, neuer Anbaumethoden sowie neuer Vermarktungsmethoden. Ebenso spiegelt sich die Offenheit der Betriebsleiter*innen, ihre Erfahrungen zum Gemüseanbau auch an andere Interessierte weiterzugeben, in der Tätigkeit des Großvaters wider, der in den 1950er Jahren andere Betriebe als Obstbau-Berater bei der Anlage von Intensiv-Obstbaukulturen unterstützt hat. Nur der Rahmen hat sich geändert: Während der Großvater in der Region als Obstbauberater tätig war, steht der Betrieb Bergmann heute im Austausch mit Betrieben aus ganz Österreich und arbeitet eng mit einem Betrieb aus Bayern bei der Entwicklung eines speziellen Anbau-Verfahrens zum ökologischen Gemüsebau zusammen. Dieses Familienmuster haben wir so beschrieben: Durch die familiäre Offenheit und Risikobereitschaft für neue Wirtschaftsweisen - die im Widerspruch zur herrschenden Praxis in der Region stehen - entstehen neue Perspektiven für die Bewirtschaftung des Betriebes, die neue Einkommensmöglichkeiten für den Betrieb schaffen und die eigene Ar- 
beitsfähigkeit und Motivation stärkt. Die Familie ist auch offen dafür, sich auch auf neue Arbeitsabläufe und neue Beziehungsgeflechte - zum Beispiel in Form von Lieferbeziehungen - einzulassen.

\section{In Bezug setzen zur erlebten Lebensgeschichte}

Erst nachdem die zentralen Themen und die Muster in der Familie aus den »objektiven « Daten des Genogramms rekonstruiert und in Form einer Fallstrukturhypothese beschrieben wurden, beschäftigen wir uns mit den subjektiven Erzählungen innerhalb der Familie zu diesen einzelnen Daten. Die Grundlage dafür sind qualitative Interviews, die wir mit den Betriebsleiter*innen geführt haben, sowie ihre Erläuterungen zu den Genogrammen. Dazu Hildenbrand: »Wir unterscheiden also zwischen einer gelebten Lebensgeschichte, welche die Genogrammdaten und die daraufbezogene Sequenz-Analyse enthält und der erlebten Lebensgeschichte, welche die biografischen Erzählungen der Klienten « enthält. ${ }^{18}$

\section{Meine berufliche Biografie und meine Rolle im Forschungsprojekt}

In der qualitativen Sozialforschung ist es Standard, sich selbst als Forscher*in $\mathrm{zu}$ verorten und den eigenen Bezug zum Forschungsfeld offenzulegen. Das Prinzip der Betroffenheit bedeutet, dass die (Selbst-)Reflexivität im Forschungsprozess für eine kritische Forschung notwendig ist. Dies bedeutet gleichzeitig, dass die eigene subjektive Perspektive der Forscherin den Forschungsprozess und die Qualität der Ergebnisse grundlegend prägt und andere Forscher aus ihrer Perspektive zu abweichenden Ergebnissen kommen können. Im Forschungsprojekt "Bio Hoch Drei«, in dessen Rahmen wir die vorliegende Publikation erarbeitet haben, führte ich die Interviews und empirischen Arbeiten »im Feld « - wie es in der Kultur- und Sozialanthropologie heißt - durch. Mit Feld ist hier nicht der Acker gemeint - 
den man auch mit mir in Verbindung bringen könnte, schließlich bin ich seit 20 Jahren als Agrarwissenschafterin tätig. Hingegen nimmt die Kultur- und Sozialanthropologie das soziale Feld in den Blick und fragt danach, wie Menschen - alleine und in Gemeinschaft - ihr Leben gestalten und ihr Zusammenleben ausrichten. Seit zwei Jahren bin ich auch als Organisationsberaterin und Beraterin für Familienbetriebe tätig und diese Tätigkeit liegt auch wie ein weites Feld vor mir, wenn ich in meine berufliche Zukunft blicke. Wenn ich den Blick in meine Vergangenheit richte, blicke ich auf fast 20 Schreibjahre zurück: Ich habe zahlreiche Fachbücher zum ökologischen Anbau von Gemüse, Obst und Kräutern verfasst. Diese Bücher sind in der »Bio-Szene« in Österreich und Deutschland sehr bekannt. Ein weiteres Tätigkeitsfeld war die Betreuung von Regionalentwicklungsprojekten für und mit Bergdörfern in Südtirol und das Konzipieren und Durchführen von Weiterbildungen für Bäuerinnen und Bauern an einer Südtiroler Fachschule für Landwirtschaft. Ich arbeite seit Abschluss meines Studiums der Agrarwissenschaften im Jahr 2000 für und mit Bauern und Bäuerinnen und durfte viele ein Stück ihres Weges begleiten, wenn es für sie gerade darum ging, neue Perspektiven für ihre Betriebe zu finden - sei es neue Kulturpflanzen anzubauen, sich Wissen zur Saatgutvermehrung anzueignen, neue Verarbeitungsmethoden oder neue Vermarktungsmethoden zu entwickeln. Auch habe ich in den letzten Jahren zahlreiche Vorträge gehalten - zum Beispiel im Jahr 2016 den Eröffnungsvortrag bei den Gemüsebau-Fachtagen des größten Österreichischen Bio-Verbandes Bio Austria. ${ }^{19}$ Ich erwähne konkret diesen Vortrag, weil ein Interview-Partner aus unserem Forschungsprojekt mich von diesem Vortrag kannte. Als ich ihn drei Jahre später in meiner Rolle als Wissenschaftlerin kontaktierte, stimmte er einem Interview sofort $\mathrm{zu}$ und rief seiner Frau zu, während ich mit ihm telefonierte: »Die Heistinger kommt zu uns. Hast du kommenden Dienstag Zeit?« Zwei andere Interviewpartner*innen kannten mich persönlich durch meine

19 Eröffnungsvortrag der Bio Austria Gemüsetage am 30.11.2015 in St. Pölten zum Thema »Bäuerliche Landwirtschaft neu denken. Abschied von Mythen und Cestalten neuer Möglichkeiten «. 
ehrenamtliche Tätigkeit im Vorstand des Vereins Arche Noah ${ }^{20}$ und durch Gespräche, die ich mit ihnen im Rahmen der Recherche für das im Jahr 2018 erschienenen Buch »Basiswissen Selbstversorgung. Individuelle und gemeinschaftliche Wege und Möglichkeiten« geführt habe. Mit meinen Gesprächspartner*innen im Niederösterreich tausche ich mich, seit sie ihren Betrieb gegründet haben, immer wieder zu Fachfragen zum Gemüsebau und zur Vermarktung aus. Einigen meiner Gesprächspartner*innen des Forschungsprojektes war ich aus diesen Zusammenhängen persönlich bekannt. Das hat einen Einfluss auf die Beziehung zwischen mir als Forscherin und den beforschten Familien und Betrieben. Die unmittelbarste Wirkung war vermutlich, dass sie recht umgehend zustimmten als Fallbeispiel für unser Forschungsprojekt Bio hoch drei zur Verfügung zu stehen. Wobei ich hier auch ergänzen möchte, dass vor allem die beiden Interviewpartner*innen, die CSA-Betriebe bewirtschaften, es gewohnt sind, dass Studierende und Forscher*innen ihre Betriebe besuchen und mit ihnen Interviews führen - über ihre Anbaumethoden und über die von ihnen gewählte Form der Vermarktung ihrer Produkte. ${ }^{21}$ Andere Reaktionen auf die Anfrage zu einem Interview waren: Freudig und rasch zu einem Gesprächstermin bereit und das Gespräch auch als Gelegenheit zur Reflexion über das eigene Tun oder als Möglichkeit zum fachlichen Austausch oder zur Inspiration wahrzunehmen. Erwähnen möchte ich an dieser Stelle auch, dass ein Betriebsleiter, den ich im Rahmen des

20 Der Verein Arche Noah mit Sitz im Niederösterreichischem Schiltern erhält in seinem Netzwerk aus Gärtner*innen und Landwirt*innen einige tausend historische Kulturpflanzensorten, die im Zuge der Industrialisierung der Landwirtschaft an Bedeutung verloren haben (www.arche-noah.at) und ist im engen Austausch mit vielen bäuerlichen Betrieben, die aus diesen Sorten wieder neue Produkte entwickeln.

21 In den letzten Jahren wurden in Österreich und Deutschland zahlreiche Forschungsprojekte und wissenschaftliche Abschlussarbeiten durchgeführt, die sich mit der Community-Supported Agriculture - auf Deutsch auch als Solidarische Landwirtschaft bezeichnet - auseinandergesetzt haben. Zum Beispiel als Case-Study im EU Horizon2020-Forschungsprojekt Simra (www.simrah2020.eu) zu sozialen Innovationen in marginalisierten ländlichen Gebieten. 
Forschungsprojektes kontaktiert hatte, es ablehnte für ein Forschungsprojekt des Landes Steiermark zur Verfügung zu stehen. Ich hatte ihn über meine offizielle E-Mail Adresse der Universität Graz kontaktiert und um ein Gespräch gebeten. Die Antwort fiel harsch aus: »Ich bin immer wieder einmal verwundert, warum es selbstverständlich ist, dass Landesoder Bundesstellen auf kostenlose Mitarbeit jener »Pioniere« zurückgreifen, denen sie davor das Leben extrem schwer gemacht haben«. In seiner Antwort wird deutlich, dass die Person mich als Forscherin als Teil des öffentlichen Bürokratiesystems sowie, dass sie sich als innovativ und Behörden nicht als Partner, sondern als Hürden der betrieblichen Innovationen wahrnimmt. Über ähnliche Erfahrungen haben Bäuerinnen und Bauern immer wieder berichtet. ${ }^{22}$ Und: Gerade unter Pionier*innen ist es weit verbreitet, dass sie mit ihrer Zeit sehr gezielt und überlegt umgehen (müssen), da die Entwicklung eines neuen Betriebszweiges, einer neuen Vermarktungs- oder Finanzierungsform sehr zeitintensiv ist. $^{23}$

\section{Schlussbemerkung}

Dieses Kapitel stellt die Methode der rekonstruktiven Genogrammarbeit vor, die eine ressourcenorientierte Methode der sinnverstehenden Sozialforschung ist. Dabei ist die rekonstruktive Genogrammarbeit eine Methode, die stark »Zwischen dem Feld« und der Theorie vermitteln

22 So berichtet eine Gesprächspartnerin: »Es war so ein Grundgedanke, dass wir da einen Stall bauen. (...). Das wurde zuerst auch genehmigt. (...) Es war die Baugenehmigung da und es ist dann an einer einzigen Person bei einer Behörde gescheitert. (...) Es waren tausende Sachen zum Umplanen, was jedes Mal etwas gekostet hat. Bis dann jeder gesagt hat, das kann man bleiben lassen. (...) Also da ist halt die Bürokratie auch blöd." (Interview am 5.7.2018) Siehe dazu auch Kapitel von Elisabeth Kosnik.

23 Von manchen Betrieben kenne ich auch die Vereinbarung, dass jemand, der über den Betrieb forschen will, auch am Betrieb mitarbeiten muss, um eine adäquate Gegenleistung für die Informationen, die für die empirische Forschung zur Verfügung gestellt wurden, im Betrieb einzubringen. 
kann, genauso wie zwischen Zukunft, Gegenwart und Vergangenheit und zwischen einem Kollektiv und Individuen, zwischen Makro- und Mikrosoziologie. Ihre Stärke ist es insbesondere, dass sie sich grundsätzlich an einem konkreten Fall orientiert: Die Grundlage der Methode ist, tatsächlich »ins Feld « zu gehen und mit den Menschen in Beziehung zu kommen. Das Kapitel »Wie aus Familiengeschichte sorgsame Landwirtschaft entsteht« führt die Analyse der vier Fallbeispiele weiter. 
